\title{
Supplementary Tables and Figures
}

Supplementary Table 1 Infants with “other” patterns of injury on MRI initial neonatal period - punctate foci in white matter.

\begin{tabular}{|c|c|c|c|c|c|c|}
\hline & $\begin{array}{l}\text { Grade } \\
\text { NE }\end{array}$ & Diffusion & $T 1$ & $T 2$ & $\begin{array}{l}\text { Follow on scan } \\
\text { (Age in } \\
\text { months) }\end{array}$ & $\begin{array}{l}\text { Outcome } \\
\text { (Age in months) }\end{array}$ \\
\hline A & 1 & Normal & $\begin{array}{l}\text { One spot R } \\
\text { deep WM }\end{array}$ & Normal & $\begin{array}{l}\text { Normal } \\
\text { (24 months) }\end{array}$ & $\begin{array}{l}\text { Mild Speech Delay } \\
\text { (17 months) }\end{array}$ \\
\hline$B$ & 2 & $\begin{array}{l}\text { Spot } L \text { ant deep } \\
\text { WM }\end{array}$ & $\begin{array}{l}\text { Spot L ant } \\
\text { deep WM }\end{array}$ & $\begin{array}{l}\text { Spot L ant } \\
\text { deep WM }\end{array}$ & $\begin{array}{l}\text { No Follow on } \\
\text { Scan }\end{array}$ & $\begin{array}{l}\text { Cognition -Normal } \\
\text { Range Dense Left } \\
\text { Erbs Palsy ( } 34 \\
\text { months) }\end{array}$ \\
\hline $\mathrm{C}$ & 2 & $\begin{array}{l}\text { R deep WM } \\
\text { Corpus } \\
\text { callosum }\end{array}$ & $\begin{array}{l}\text { Bright spot } \\
\text { R deep WM }\end{array}$ & $\begin{array}{l}\text { Dark spot } \\
\text { R deep WM }\end{array}$ & $\begin{array}{l}\text { No Follow on } \\
\text { Scan }\end{array}$ & $\begin{array}{l}\text { Hearing Impairment, } \\
\text { Mild Learning } \\
\text { disability (59 months) }\end{array}$ \\
\hline$D$ & 2 & & $\begin{array}{l}\text { Tiny foci } L \\
\text { post deep } \\
\text { WM }\end{array}$ & $\begin{array}{l}\text { Tiny foci } L \\
\text { post deep WM }\end{array}$ & $\begin{array}{l}\text { No Follow on } \\
\text { Scan }\end{array}$ & $\begin{array}{l}\text { Normal } \\
\text { (64 months) }\end{array}$ \\
\hline$E$ & 1 & Foci L deep WM & $\begin{array}{l}\text { Foci L deep } \\
\text { WM }\end{array}$ & $\begin{array}{l}\text { Foci L deep } \\
\text { WM }\end{array}$ & $\begin{array}{l}2 \text { Focal areas } \\
\text { abnormal signal } \\
\text { WM (19 months) }\end{array}$ & Normal (49 Months) \\
\hline $\mathrm{F}$ & 2 & $\begin{array}{l}\text { Foci deep white } \\
\text { matter BL, } \\
\text { PLIC BL }\end{array}$ & Normal & $\begin{array}{l}\text { Small deep } \\
\text { WM spot }\end{array}$ & & $\begin{array}{l}\text { Autism, ADHD } \\
\text { (102 Months) }\end{array}$ \\
\hline G & 2 & $\begin{array}{l}\text { Foci deep WM } \\
\text { BL anterior and } \\
\text { posterior }\end{array}$ & $\begin{array}{l}\text { Foci deep } \\
\text { WM } \\
\text { BL anterior } \\
\text { and posterior }\end{array}$ & $\begin{array}{l}\text { Foci deep WM } \\
\text { BL anterior } \\
\text { and posterior }\end{array}$ & $\begin{array}{l}\text { Focal areas } \\
\text { abnormal signal } \\
\text { WM ( } 27 \text { months) }\end{array}$ & $\begin{array}{l}\text { Mild impairments } \\
\text { concentration and } \\
\text { attention (49 Months) }\end{array}$ \\
\hline $\mathrm{H}$ & 2 & $\begin{array}{l}\text { Anterior and } \\
\text { posterior corpus } \\
\text { callosum }\end{array}$ & $\begin{array}{l}2 \text { small foci } \\
\text { adjacent to } \\
\text { lateral } \\
\text { ventricle }\end{array}$ & $\begin{array}{l}2 \text { small foci } \\
\text { adjacent to } \\
\text { lateral } \\
\text { ventricle }\end{array}$ & $\begin{array}{l}\text { Focal areas } \\
\text { abnormal signal } \\
\text { WM BL } \\
\text { (71 months) }\end{array}$ & $\begin{array}{l}\text { Impairments Memory } \\
\text { and Language } \\
\text { Epilepsy } \\
\text { (91 Months) }\end{array}$ \\
\hline & $\mathrm{mat}$ & $\mathrm{R}=$ right & $\mathrm{BL}=$ bilate & & & \\
\hline
\end{tabular}


Supplementary Table 2 Infants with “other” patterns of injury on MRI initial neonatal period - not punctate foci.

\begin{tabular}{|c|c|c|c|c|c|c|}
\hline & $\begin{array}{l}\text { Grade } \\
\text { NE }\end{array}$ & Diffusion & T1 & T2 & $\begin{array}{l}\text { Follow on scan } \\
\text { (Age in months) }\end{array}$ & $\begin{array}{l}\text { Outcome } \\
\text { (Age in } \\
\text { months) }\end{array}$ \\
\hline$I$ & 1 & $\begin{array}{l}\text { Focal } \\
\text { area } \\
\text { abnormal } \\
\text { diffusion } \\
\mathrm{L} \\
\text { thalamus }\end{array}$ & $\begin{array}{l}\text { Focal high signal L } \\
\text { thalamus }\end{array}$ & $\begin{array}{l}\text { Focal low signal L } \\
\text { thalamus }\end{array}$ & $\begin{array}{l}\text { Focal abnormality } \\
\text { signal L thalamus } \\
\text { (27 months) }\end{array}$ & $\begin{array}{l}\text { Normal } \\
\text { Range (52 } \\
\text { months) }\end{array}$ \\
\hline $\mathrm{J}$ & 2 & Normal & $\begin{array}{l}\text { BL Thalami LTF } \\
\text { L Posterior parietal foci } \\
\text { BL abn PLIC }\end{array}$ & Thalami & $\begin{array}{l}\text { Abnormal signal } \\
\text { BL deep WM. } \\
\text { Focal area } \\
\text { abnormal signal in } \\
\text { antero-medial } \\
\text { thalami BL (18 } \\
\text { months) }\end{array}$ & $\begin{array}{l}\text { Mild } \\
\text { Dyskinetic } \\
\text { CP } \\
\text { Normal } \\
\text { Cognition } \\
\text { (36months) }\end{array}$ \\
\hline K & 2 & $\begin{array}{l}\text { Abnormal } \\
\text { diffusion } \\
\text { BL } \\
\text { occipital } \\
\text { and } \\
\text { parietal }\end{array}$ & Abnormal L PLIC & $\begin{array}{l}\text { Abnormal L PLIC } \\
\text { and superior thalamus }\end{array}$ & $\begin{array}{l}\text { No Follow on } \\
\text { Scan }\end{array}$ & $\begin{array}{l}\text { Normal } \\
\text { Range (78 } \\
\text { months) }\end{array}$ \\
\hline $\mathrm{L}$ & 2 & $\begin{array}{l}\text { Abnormal } \\
\text { signal } \\
\text { parietal } \\
\text { and } \\
\text { occipital } \\
\text { cortex } \\
\text { and } \\
\text { thalami }\end{array}$ & $\begin{array}{l}\text { BL posterior cerebral, } \\
\text { BL PLIC } \\
\text { Large subdural } \\
\text { haematoma }\end{array}$ & $\begin{array}{l}\text { BL posterior cerebral, } \\
\text { BL PLIC } \\
\text { Large subdural } \\
\text { haematoma }\end{array}$ & $\begin{array}{l}\text { Enlarged } \\
\text { ventricles, WM } \\
\text { volume loss BL } \\
\text { Encephalomalacia } \\
\text { BL parietal. BL } \\
\text { abnormal signal } \\
\text { cerebellar } \\
\text { hemispheres. } \\
\text { (10.7 months) }\end{array}$ & $\begin{array}{l}\text { Severe } \\
\text { spastic } \\
\text { quadriplegia, } \\
\text { Epilepsy, } \\
\text { Non-verbal. } \\
\text { Blind } \\
\text { (70 months) }\end{array}$ \\
\hline$M$ & 1 & Normal & $\begin{array}{l}\text { Cysts in deep WM (L } \\
\text { anterior \& posterior; R } \\
\text { anterior) } \\
\text { Subduralposteriorfossa, } \\
\text { abnormal signal L } \\
\text { thalamus }\end{array}$ & $\begin{array}{l}\text { Cysts in deep WM (L } \\
\text { anterior \& posterior; R } \\
\text { anterior) } \\
\text { Subduralposteriorfossa, } \\
\text { abnormal signal L } \\
\text { thalamus }\end{array}$ & $\begin{array}{l}\text { Loss of volume } \\
\text { deep WM L } \\
\text { posterior. } \\
\text { Abnormal high } \\
\text { signal in } \\
\text { posterolateral L } \\
\text { thalamus \& PLIC } \\
\text { (20.7 months) }\end{array}$ & $\begin{array}{l}\text { Hemiplegia } \\
\text { Normal } \\
\text { range } \\
\text { cognition }\end{array}$ \\
\hline $\mathrm{N}$ & 2 & $\begin{array}{l}\text { Abnormal } \\
\text { diffusion } \\
\mathrm{R} \\
\text { occipital } \\
\text { and L } \\
\text { temporal } \\
\text { lobe }\end{array}$ & $\begin{array}{l}\text { Abnormal signal } R \\
\text { occipital and } L \text { temporal } \\
\text { lobe }\end{array}$ & $\begin{array}{l}\text { Abnormal signal } R \\
\text { occipital and } L \text { temporal } \\
\text { lobe }\end{array}$ & $\begin{array}{l}\text { Focal areas } \\
\text { abnormal signal } \\
\text { posterior deep } \\
\text { WM BL ( } 26 \\
\text { months) }\end{array}$ & $\begin{array}{l}\text { Moderate to } \\
\text { Severe } \\
\text { hearing } \\
\text { Impairment, } \\
\text { Autism }\end{array}$ \\
\hline \multicolumn{7}{|c|}{$\mathrm{R}=$ right } \\
\hline
\end{tabular}


Supplementary Table 3 Detailed Injury Score and Outcome

\begin{tabular}{|c|c|c|c|c|c|c|c|c|c|c|c|}
\hline \multicolumn{12}{|c|}{ Detailed Injury Score } \\
\hline & \multirow{2}{*}{$\begin{array}{l}\text { PLIC } \\
\text { Affected } \\
\text { Yes }\end{array}$} & \multicolumn{3}{|c|}{ BGT Score } & \multicolumn{3}{|c|}{ WS score } & \multicolumn{4}{|c|}{ Total Score } \\
\hline & & 0 & $1-2$ & $3-4$ & 0 & $1-2$ & $4-5$ & 0 & $1-3$ & $4-5$ & $6-9$ \\
\hline Normal & 0 & 12 & 1 & 1 & 11 & 3 & 0 & 10 & 4 & 0 & 0 \\
\hline Abnormal & 21 & 14 & 3 & 18 & 21 & 3 & 10 & 11 & 7 & 7 & 9 \\
\hline Death & 5 & 0 & 0 & 7 & 2 & 0 & 5 & 0 & 0 & 2 & 5 \\
\hline Cerebral Palsy & 9 & 1 & 0 & 10 & 6 & 0 & 4 & 0 & 3 & 4 & 3 \\
\hline Epilepsy(Isolated) & 0 & 0 & 0 & 1 & 0 & 0 & 1 & 0 & 0 & 0 & 1 \\
\hline Autism & 1 & 1 & 0 & 0 & 1 & 0 & 0 & 1 & 0 & 0 & 0 \\
\hline $\begin{array}{l}\text { Low Assessment } \\
\text { Scores }\end{array}$ & 17 & 13 & 3 & 3 & 13 & 3 & 2 & 11 & 4 & 1 & 2 \\
\hline No assessment & 2 & 22 & 2 & 0 & 19 & 3 & 3 & 17 & 5 & 2 & 1 \\
\hline Total & 23 & 48 & 6 & 21 & 51 & 9 & 14 & 38 & 16 & 9 & 11 \\
\hline \multicolumn{12}{|l|}{ BSITD 3} \\
\hline Normal & 0 & 12 & 1 & 1 & 11 & 3 & 0 & 10 & 4 & 0 & 0 \\
\hline Clinical/borderline & 2 & 4 & 1 & 1 & 4 & 1 & 1 & 4 & 1 & 0 & 1 \\
\hline Total & 2 & 16 & 2 & 2 & 15 & 4 & 1 & 14 & 5 & 0 & 1 \\
\hline \multicolumn{12}{|l|}{ NEPSY 2} \\
\hline Normal & 1 & 5 & 0 & 0 & 4 & 1 & 0 & 4 & 1 & 0 & 0 \\
\hline Clinical/borderline & 4 & 5 & 2 & 2 & 6 & 1 & 1 & 4 & 2 & 1 & 1 \\
\hline Total & 5 & 10 & 2 & 2 & 10 & 2 & 1 & 8 & 3 & 1 & 1 \\
\hline \multicolumn{12}{|l|}{ Movement ABC } \\
\hline Normal & 3 & 7 & 1 & 1 & 7 & 2 & 0 & 6 & 2 & 1 & 0 \\
\hline Clinical/borderline & 1 & 4 & 1 & 1 & 4 & 0 & 1 & 3 & 1 & 0 & 1 \\
\hline Total & 4 & 11 & 2 & 2 & 11 & 2 & 1 & 9 & 3 & 1 & 1 \\
\hline \multicolumn{12}{|l|}{$\begin{array}{c}\text { Child Behaviour } \\
\text { Checklist }\end{array}$} \\
\hline Normal & 1 & 4 & 0 & 0 & 3 & 0 & 0 & 3 & 0 & 0 & 0 \\
\hline Clinical/borderline & 4 & 6 & 2 & 2 & 7 & 2 & 1 & 5 & 3 & 1 & 1 \\
\hline Total & 5 & 10 & 2 & 2 & 10 & 2 & 1 & 8 & 3 & $\frac{1}{1}$ & 1 \\
\hline \multicolumn{12}{|l|}{ BRIEF } \\
\hline Normal & 0 & 1 & 0 & 0 & 1 & 0 & 0 & 1 & 0 & 0 & 0 \\
\hline Clinical/borderline & 2 & 7 & 1 & 1 & 6 & 1 & 1 & 5 & 2 & 0 & 1 \\
\hline Total & 2 & 8 & 1 & 1 & 7 & 1 & 1 & 6 & 2 & 0 & 1 \\
\hline
\end{tabular}




\section{Supplementary Figure 1. Imaging and Outcome.}

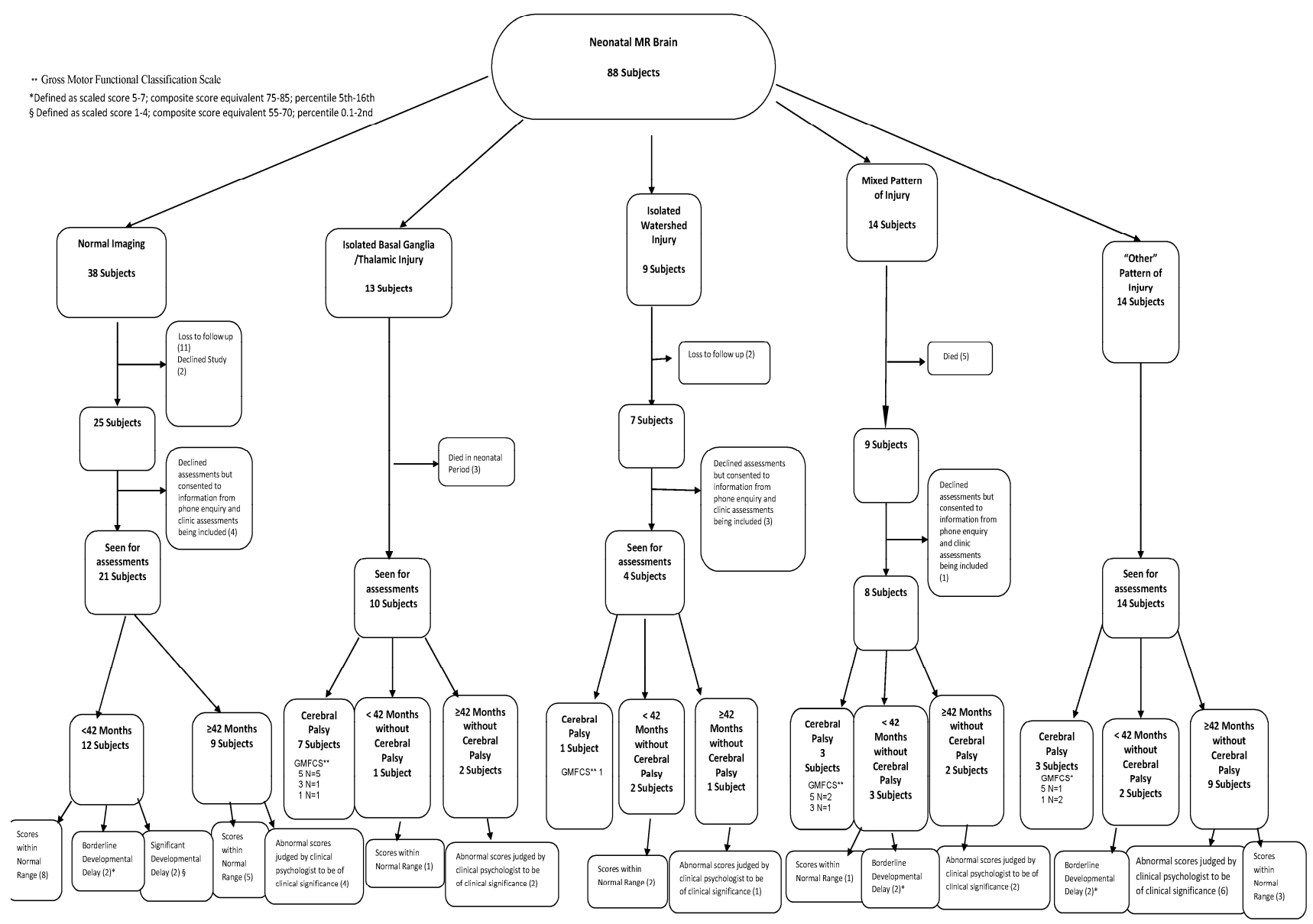


Supplementary Figure 2. Basal ganglia / thalamic pattern of injury.MRI day 5.Apparent diffusion coefficient (ADC)* image showing darker area consistent with restricted diffusion

(a) In thalami and basal ganglia bilaterally

(b) In cortex of central sulcus area bilaterally.

(c) Another baby MRI day 4, restricted diffusion in both hippocampal areas (arrows).

(d) Another baby MRI day 6. T1 image shows abnormal high T1 signal in both lentiform nuclei and in the lateral nuclei of the thalami bilaterally. There is loss of the normal high signal of the posterior limb of the internal capsule (PLIC) bilaterally.
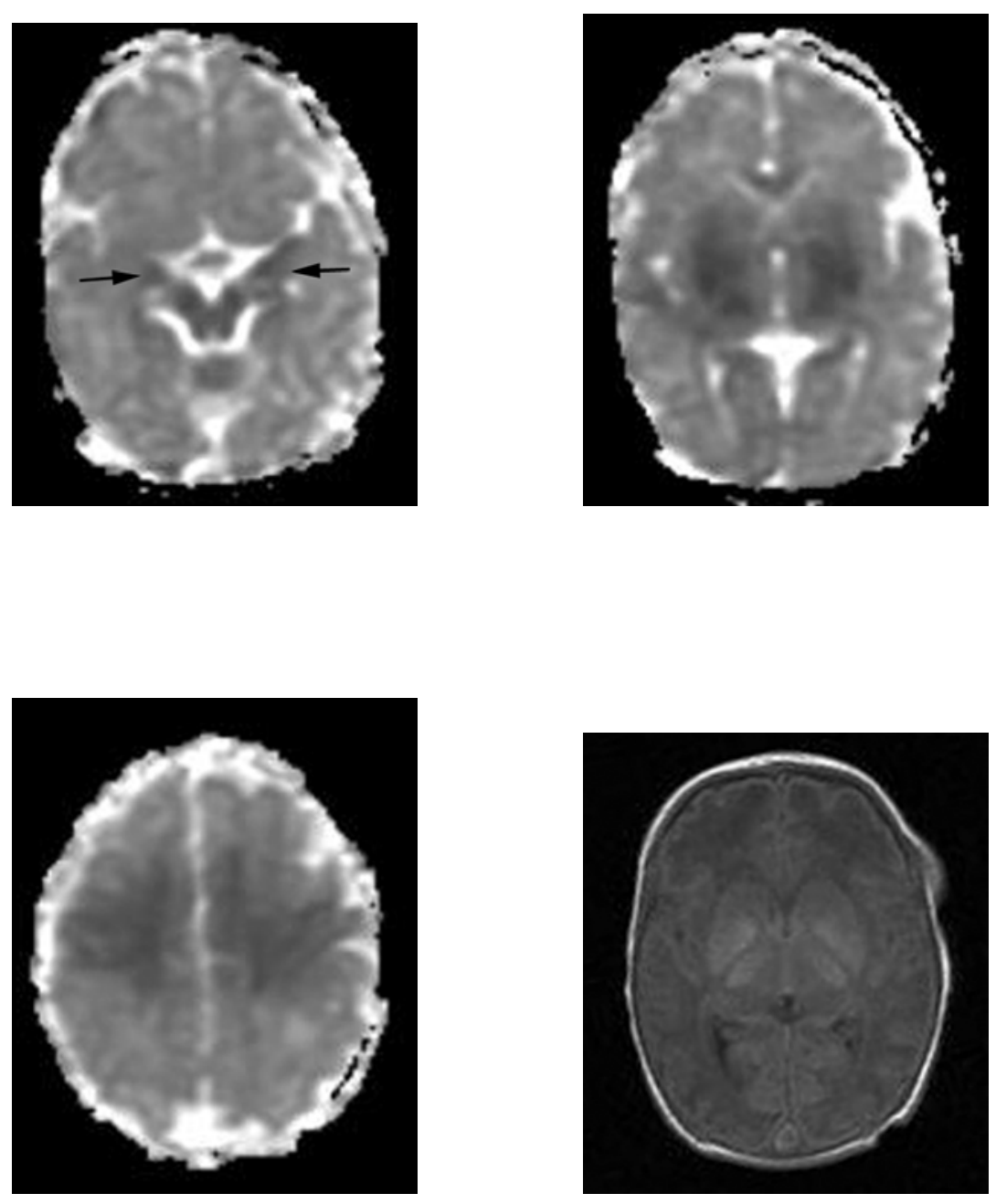
Supplementary Figure 3. Watershed pattern of injury. MRI day 4, diffusion weighted images show abnormal high signal in

(a) Posterior watershed areas bilaterally,

(b) Anterior watershed area anteriorly.
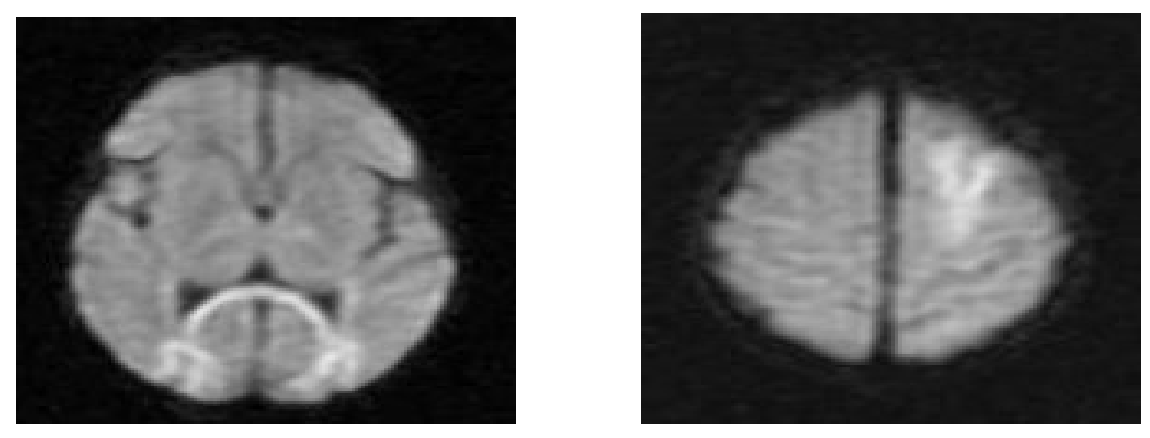

Supplementary Figure 4. Mixed pattern of injury. MRI day 5.

Apparent diffusion coefficient (ADC)*image showing darker area consistent with restricted diffusion in both thalami and caudate nuclei as well as in posterior and anterior watershed areas and in the anterior and posterior corpus callosum.

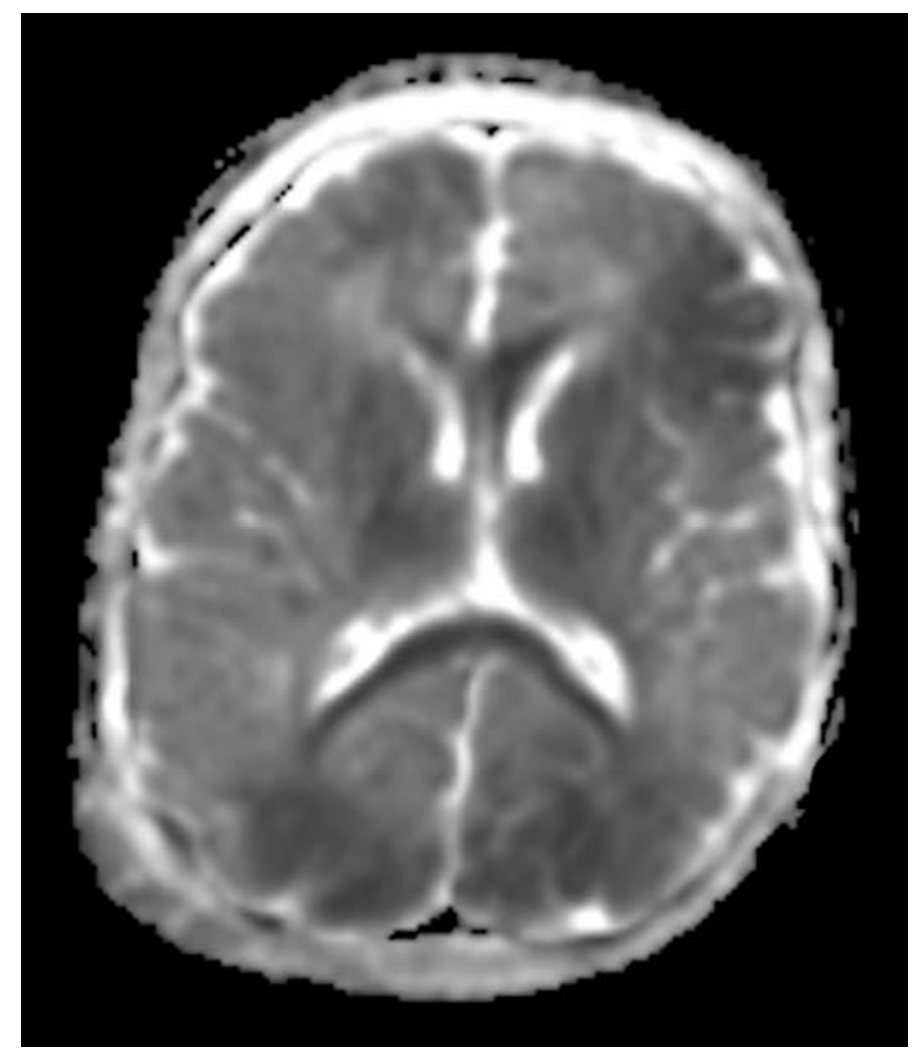


Supplementary Figure 5. Cerebellar involvement. MRI day 5.

(a) T2 images showing brightness and swelling consistent with oedema in both cerebellar hemispheres as well as in both temporal lobes and in the brain stem.

(b) Normal T2 image at same level in another baby for comparison with (a)
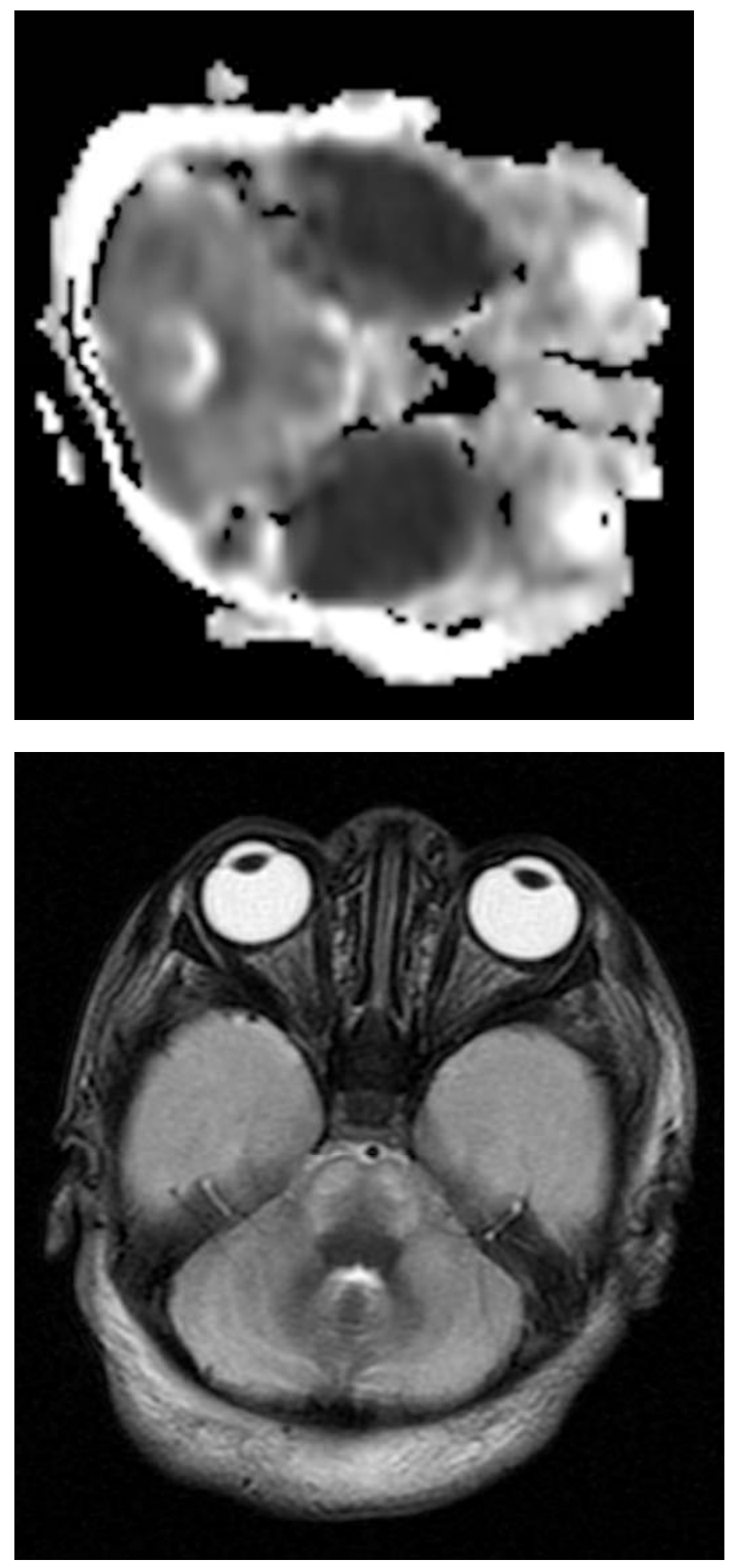
Supplementary Figure 6. Other pattern of injury. MRI day 7 shows abnormal signal in deep white matter adjacent to body of left lateral ventricle,

(a) Focus of high T2 signal with low signal surround (arrow)

(b) ADC* image shows low signal in the affected area consistent with restricted diffusion (arrow).

*ADC images chosen for better demonstration of acute ischaemic injury although brain structure less sharply defined compared to T1/T2 images.1.
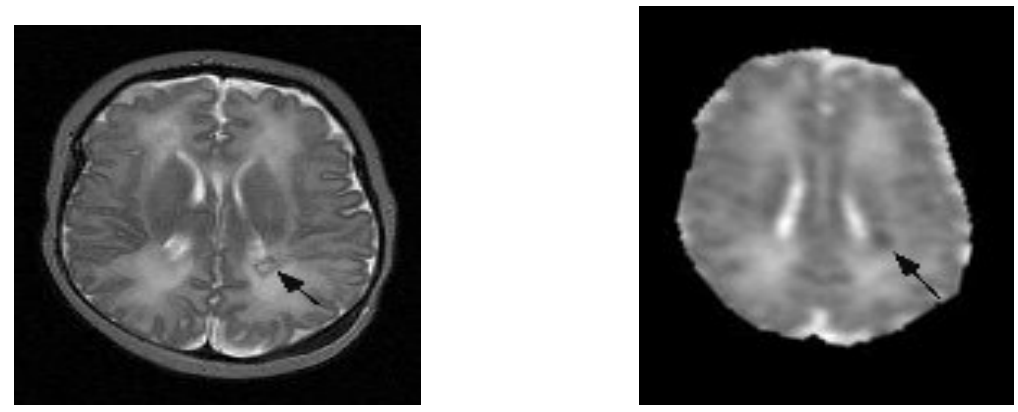